PROCEEDINGS OF THE

AMERICAN MATHEMATICAL SOCIETY

Volume 136, Number 12, December 2008, Pages 4313-4320

S 0002-9939(08)09417-3

Article electronically published on June 25, 2008

\title{
A RIGIDITY THEOREM FOR HOLOMORPHIC GENERATORS ON THE HILBERT BALL
}

\author{
MARK ELIN, MARINA LEVENSHTEIN, SIMEON REICH, AND DAVID SHOIKHET
}

(Communicated by N. Tomczak-Jaegermann)

\begin{abstract}
We present a rigidity property of holomorphic generators on the open unit ball $\mathbb{B}$ of a Hilbert space $H$. Namely, if $f \in \operatorname{Hol}(\mathbb{B}, H)$ is the generator of a one-parameter continuous semigroup $\left\{F_{t}\right\}_{t>0}$ on $\mathbb{B}$ such that for some boundary point $\tau \in \partial \mathbb{B}$, the admissible limit $K$ - $\lim _{z \rightarrow \tau} \frac{f(x)}{\|x-\tau\|^{3}}=0$, then $f$ vanishes identically on $\mathbb{B}$.
\end{abstract}

Let $H$ be a complex Hilbert space with inner product $\langle\cdot, \cdot\rangle$ and induced norm $\|\cdot\|$. If $H$ is finite dimensional, we will identify $H$ with $\mathbb{C}^{n}$. We denote by $\operatorname{Hol}(D, E)$ the set of all holomorphic mappings on a domain $D \subset H$ which map $D$ into a subset $E$ of $H$, and put $\operatorname{Hol}(D):=\operatorname{Hol}(D, D)$.

We are concerned with the problem of finding conditions for a mapping $F \in$ $\operatorname{Hol}(D, E)$ to coincide identically with a given holomorphic mapping on $D$ when they behave similarly in a neighborhood of a boundary point $\tau \in \partial D$.

A number of basic results in this direction are due to D. M. Burns and S. G. Krantz 6]. They establish conditions at a boundary point for a holomorphic self-mapping $F$ of the open unit disk $\Delta:=\{z \in \mathbb{C}:|z|<1\}$ to coincide with the identity mapping (see Proposition 1 below). Then they generalize this fact to the $n$-dimensional case: for holomorphic self-mappings of the open unit ball (see Proposition 3 below) and of strongly pseudoconvex domains in $\mathbb{C}^{n}$. Further developments of this theme are presented by X. J. Huang in [15], where he obtains similar results for weakly pseudoconvex domains. More recently, L. Baracco, D. Zaitsev and G. Zampieri [3] have proved local boundary rigidity theorems for mappings defined only on one side as germs at a boundary point, and extended their results from boundaries of domains to submanifolds of higher codimension. More higher-dimensional results can be found, for instance, in [2] and [11.

In this paper we present a rigidity theorem for holomorphic generators on the open unit ball $\mathbb{B}$ of a Hilbert space $H$ which generalizes the analogous theorem for the one-dimensional case [8, 17, 7] and properly contains the above-mentioned Burns-Krantz theorem for the open unit ball in $\mathbb{C}^{n}$.

Received by the editors July 30, 2007, and, in revised form, October 20, 2007.

2000 Mathematics Subject Classification. Primary 30C45, 30D05, 46T25, 47H20.

Key words and phrases. Angular limit, Hilbert ball, holomorphic generator, $K$-limit, oneparameter continuous semigroup, rigidity.

The third author was partially supported by the Fund for the Promotion of Research at the Technion and by the Technion President's Research Fund.

All the authors thank the referee for several helpful comments and suggestions.

(C)2008 American Mathematical Society 
We begin by recalling the result of D. M. Burns and S. G. Krantz [6] for holomorphic self-mappings of the open unit disk $\Delta$.

Proposition 1. Let $F \in \operatorname{Hol}(\Delta)$. If the unrestricted limit

$$
\lim _{z \rightarrow 1} \frac{F(z)-z}{(z-\tau)^{3}}=0
$$

for some $\tau \in \partial \Delta$, then $F \equiv I$ on $\Delta$.

This assertion also holds when the unrestricted limit is replaced with the angular one (see [22] and [5]). Recall that a function $f \in \operatorname{Hol}(\Delta, \mathbb{C})$ has an angular limit $L:=\angle \lim _{z \rightarrow \tau} f(z)$ at a point $\tau \in \partial \Delta$ if $f(z) \rightarrow L$ as $z \rightarrow \tau$ in each nontangential approach region

$$
\Gamma_{k}(\tau):=\left\{z \in \Delta: \frac{|z-\tau|}{1-|z|}<k\right\}, k>1 .
$$

In this case it is convenient to set $f(\tau):=\angle \lim _{z \rightarrow \tau} f(z)$. Moreover, in a similar way, one defines the angular derivative of $f$ at $\tau \in \partial \Delta$ by $f^{\prime}(\tau):=\angle \lim _{z \rightarrow \tau} \frac{f(z)-f(\tau)}{z-\tau}$.

A point $\tau \in \bar{\Delta}$ is a fixed point of $F \in \operatorname{Hol}(\Delta)$ if either $F(\tau)=\tau$, where $\tau \in \Delta$, or $\lim _{r \rightarrow 1^{-}} F(r \tau)=\tau$, where $\tau \in \partial \Delta=\{z:|z|=1\}$. If $F$ is not an automorphism of $\Delta$ with an interior fixed point, then by the classical Schwarz-Pick lemma and the Julia-Wolff-Carathéodory theorem, there is a unique fixed point $\tau \in \bar{\Delta}$ such that for each $z \in \Delta, \lim _{n \rightarrow \infty} F_{n}(z)=\tau$, where the $n$-th iteration $F_{n}$ of $F$ is defined by $F_{1}=F, F_{n}=F \circ \stackrel{n \rightarrow \infty}{\circ} F_{n-1}, n=2,3, \ldots$. This point is called the Denjoy-Wolff point of $F$. Moreover, a boundary fixed point $\tau \in \partial \Delta$ of $F$ is its Denjoy-Wolff point if and only if $F^{\prime}(\tau) \in(0,1]$.

A rigidity result for generators of one-parameter continuous semigroups on $\Delta$ (see Proposition 2 below) has been proved in 8 and [17. To formulate it, we first recall the definitions of these notions.

Let $D \subset H$ be a domain in the Hilbert space $H$. We say that a family $S=\left\{F_{t}\right\}_{t \geq 0} \subset \operatorname{Hol}(D)$ is a one-parameter continuous semigroup on $D$ (a semigroup, for short) if

(i) $F_{t}\left(F_{s}(z)\right)=F_{t+s}(z)$ for all $t, s \geq 0$ and all $z \in D$, and

(ii) $\lim _{t \rightarrow 0^{+}} F_{t}(z)=z$ for all $z \in D$.

A semigroup $S=\left\{F_{t}\right\}_{t \geq 0} \subset \operatorname{Hol}(D)$ is said to be generated if for each $z \in D$, there exists the strong limit

$$
\lim _{t \rightarrow 0^{+}} \frac{1}{t}\left[z-F_{t}(z)\right]=f(z) .
$$

In this case the mapping $f: D \mapsto H$ is called the (infinitesimal) generator of $S$.

A well-known representation of generators on $\Delta$ is due to E. Berkson and H. Porta [4, namely:

A function $f \in \operatorname{Hol}(\Delta, \mathbb{C})$ is a generator if and only if there are a point $\tau \in \bar{\Delta}$ and a function $p \in \operatorname{Hol}(\Delta, \mathbb{C})$ with $\operatorname{Re} p(z) \geq 0$ for all $z \in \Delta$ such that

$$
f(z)=(z-\tau)(1-\bar{\tau} z) p(z), \quad z \in \Delta .
$$

This point $\tau$ is the common Denjoy-Wolff point of the semigroup generated by $f$.

The following rigidity result for generators has been proved in [8] and [17]. 
Proposition 2. Let $g \in \operatorname{Hol}(\Delta, \mathbb{C})$ be the generator of a one-parameter continuous semigroup. Suppose that

$$
\angle \lim _{z \rightarrow 1} \frac{g(z)}{|z-1|^{3}}=0
$$

Then $g \equiv 0$ in $\Delta$.

Here we take this opportunity to present a completely different proof of this assertion.

Proof. Suppose that $g$ does not vanish identically on $\Delta$. Condition (2) implies that $\tau=1$ is the Denjoy-Wolff point of the semigroup generated by $g$ (see Lemma 3 in [10]). So, $g$ has no null point in $\Delta$ (see Theorem 1 in [10]). Consequently, $g$ can be represented by the Berkson-Porta formula

$$
g(z)=-(1-z)^{2} p(z), \quad z \in \Delta,
$$

where $p$ is a holomorphic function of nonnegative real part which does not vanish in $\Delta$.

Consider the function

$$
g_{1}(z):=\frac{-z}{(1-z)^{2}} \cdot g(z)=z p(z), \quad z \in \Delta .
$$

This function is the holomorphic generator of a semigroup on $\Delta$ with its DenjoyWolff point at zero.

However, the equality

$$
\angle \lim _{z \rightarrow 1} \frac{g_{1}(z)}{z-1}=\angle \lim _{z \rightarrow 1} \frac{-z}{(1-z)^{3}} \cdot g(z)=0
$$

implies that $g_{1}(1)=0$ and $g_{1}^{\prime}(1)=0$. Therefore $\tau=1$, too, is the DenjoyWolff point of the semigroup generated by $g_{1}$ (again by Lemma 3 in [10]). The contradiction we have reached proves that $g \equiv 0$ on $\Delta$.

As we have already mentioned above, D. M. Burns and S. G. Krantz generalize their one-dimensional result for holomorphic self-mappings of $\Delta$ (Proposition 1) to the open unit ball $\mathbb{B}:=\left\{x \in \mathbb{C}^{n}:\|x\|<1\right\}$, where $\|x\|=\sqrt{\left|x_{1}\right|^{2}+\left|x_{2}\right|^{2}+\ldots+\left|x_{n}\right|^{2}}$.

Proposition 3 (see [6]). Let $\mathbb{B} \subset \mathbb{C}^{n}$ be the open unit ball. Let $\Phi: \mathbb{B} \rightarrow \mathbb{B}$ be a holomorphic mapping of the ball to itself such that

$$
\Phi(x)=\mathbf{1}+(x-\mathbf{1})+O\left(\|x-\mathbf{1}\|^{4}\right)
$$

as $x \rightarrow \mathbf{1}$. (Here $\mathbf{1}$ denotes the distinguished boundary point $\mathbf{1}=(1,0, \ldots, 0)$ of the ball.) Then $\Phi(x)=x$ on the ball.

At this juncture, a natural question arises: does the rigidity result for generators (Proposition 2) admit an analogous generalization to the open unit balls of either $\mathbb{C}^{n}$ or a Hilbert space $H$ ? The following theorem gives an affirmative answer to this question. Moreover, we show that it is sufficient to consider the $K$-limit instead of the unrestricted one in the assumption of the theorem.

Let $\mathbb{B}$ be the open unit ball of the Hilbert space $H$. For $\alpha>1$, we denote by

$$
D_{\alpha}(\tau):=\left\{x \in \mathbb{B}:|1-\langle x, \tau\rangle|<\frac{\alpha}{2}\left(1-\|x\|^{2}\right)\right\}
$$


the Korányi approach regions at $\tau \in \partial \mathbb{B}$ and say that a mapping $f: \mathbb{B} \mapsto H$ has a $K$-limit $M$ at $\tau$ if it tends to $M$ along every curve ending at $\tau$ and lying in a Korányi region $D_{\alpha}(\tau)$.

Theorem. Let $f \in \operatorname{Hol}(\mathbb{B}, H)$ be the generator of a one-parameter continuous semigroup on $\mathbb{B}$. If for some $\tau \in \partial \mathbb{B}$, the $K$-limit

$$
K-\lim _{x \rightarrow \tau} \frac{f(x)}{\|x-\tau\|^{3}}=0
$$

then $f \equiv 0$ on $\mathbb{B}$.

Proof. We prove this assertion by reduction to the one-dimensional case. Namely, we consider the restriction of the orthogonal projection of an appropriate modification of the generator $f$ to a one-dimensional disk touching $\mathbb{B}$ at the point $\tau \in \partial \mathbb{B}$. To this end, we fix a point $y \in \mathbb{B}$ and define the mapping

$$
M_{y}(x):=\frac{y-P_{y} x-s Q_{y} x}{1-\langle x, y\rangle}, \quad x \in \mathbb{B},
$$

where $P_{y}$ is the orthogonal projection of $H$ onto the subspace generated by $y\left(P_{0} \equiv 0\right.$ and $P_{y} x=\frac{\langle x, y\rangle}{\|y\|^{2}} y$ for $\left.y \neq 0\right), Q_{y}=I-P_{y}$ and $s=\sqrt{1-\|y\|^{2}}$. This mapping is an automorphism of $\mathbb{B}$ satisfying $M_{y}^{-1}=M_{y}$ (cf. p. 98 in [12] and p. 25 in [20]).

Denote by $U_{y}$ a unitary operator on $\mathbb{B}$ such that $U_{y} \tau=M_{y} \tau$. Then the mapping $m:=M_{y} \circ U_{y}$ is an automorphism of $\mathbb{B}$ which satisfies $m(\tau)=\tau$ and $m(0)=y$.

Obviously, $m$ is a biholomorphism of $\mathbb{B}$ onto $\mathbb{B}$. Therefore, by Lemma 3.7.1 on p. 30 of [9], the mapping

$$
f_{m}(w)=\left[m^{\prime}(w)\right]^{-1} f(m(w)), \quad w \in \mathbb{B},
$$

is also a holomorphic generator on $\mathbb{B}$.

Substituting

$$
\left[m^{\prime}(w)\right]^{-1}=\left[m^{-1}(x)\right]_{x=m(w)}^{\prime}=U_{y}^{*} M_{y}^{\prime}(m(w))
$$

in (4), we have

$$
f_{m}(w)=U_{y}^{*} M_{y}^{\prime}(m(w)) f(m(w)), \quad w \in \mathbb{B} .
$$

Now we define a holomorphic function $g$ on the unit disk $\Delta$ of the complex plane $\mathbb{C}$ by

$$
g(z):=\left\langle f_{m}(z \tau), \tau\right\rangle, \quad z \in \Delta .
$$

This function $g$ is a holomorphic generator on $\Delta$. To see this, note that by the Theorem in [1], the generator $f_{m}$ satisfies the inequality

$$
\operatorname{Re}\left\langle f_{m}(x)-\left(1-\|x\|^{2}\right) f_{m}(0), x\right\rangle \geq 0 \text { for all } x \in \mathbb{B} .
$$

In particular, for $x=z \tau$, where $z \in \Delta$,

$$
\operatorname{Re}\left(\left\langle f_{m}(z \tau), \tau\right\rangle \bar{z}\right) \geq\left(1-|z|^{2}\right) \operatorname{Re}\left(\left\langle f_{m}(0), \tau\right\rangle \bar{z}\right) ;
$$

i.e.,

$$
\operatorname{Re}(g(z) \bar{z}) \geq\left(1-|z|^{2}\right) \operatorname{Re}(g(0) \bar{z}) \quad \text { for all } \quad z \in \Delta,
$$

and, consequently, by the same theorem (see [1]), $g$ is indeed a holomorphic generator on $\Delta$. (We remark in passing that this also follows from the characterization of generators in terms of their $\rho$-monotonicity [18, 16].) 
We claim that under our assumptions, $g \equiv 0$ on $\Delta$. Indeed,

$$
\begin{aligned}
g(z) & =\left\langle U_{y}^{*} M_{y}^{\prime}(m(z \tau)) f(m(z \tau)), \tau\right\rangle=\left\langle M_{y}^{\prime}(m(z \tau)) f(m(z \tau)), U_{y} \tau\right\rangle \\
& =\left\langle f(m(z \tau)),\left[M_{y}^{\prime}(m(z \tau))\right]^{*} U_{y} \tau\right\rangle, \quad z \in \Delta,
\end{aligned}
$$

and, consequently,

$$
\begin{aligned}
\frac{g(z)}{|z-1|^{3}} & =\frac{1}{|z-1|^{3}}\left\langle f(m(z \tau)),\left[M_{y}^{\prime}(m(z \tau))\right]^{*} U_{y} \tau\right\rangle \\
& =\frac{\|m(z \tau)-\tau\|^{3}}{|z-1|^{3}}\left\langle\frac{f(m(z \tau))}{\|m(z \tau)-\tau\|^{3}},\left[M_{y}^{\prime}(m(z \tau))\right]^{*} U_{y} \tau\right\rangle .
\end{aligned}
$$

Note that each automorphism $h$ of $\mathbb{B}$ is the restriction to $\mathbb{B}$ of a holomorphic mapping defined either on the larger ball $B(0, R)$ centered at zero of radius $R=$ $\frac{1}{\left\|h^{-1}(0)\right\|}$ if $h(0) \neq 0$ or on all of $H$ if $h$ fixes the origin. So, $M_{y}$ and $m$ are, in fact, holomorphic mappings defined either on the open ball $B(0, R)$ of radius $R=\frac{1}{\|y\|}>1$ if $y \neq 0$ or on $H$ if $y=0$. Hence the first factor on the right-hand side of equality (8) has a finite limit as $z \rightarrow 1$, and so has the second factor of the inner product.

Now we show that the first factor of the last inner product in (8) tends to zero as $z \rightarrow 1$ nontangentially in $\Delta$.

For $z$ close enough to 1 in the nontangential approach region

$$
\Gamma_{k}=\left\{z \in \Delta: \frac{|z-1|}{1-|z|}<k\right\}, \quad k>1,
$$

$m(z \tau)$ belongs to the Korányi region $D_{\alpha}(\tau)$ whenever $\alpha>k$. Indeed, it can be shown by direct calculations that the function $m$ satisfies the equality

$$
\frac{|1-\langle m(z \tau), \tau\rangle|^{2}}{1-\|m(z \tau)\|^{2}}=L \frac{|1-z|^{2}}{1-|z|^{2}}, \quad z \in \Delta,
$$

where

$$
L:=\left.\frac{d}{d z}\langle m(z \tau), \tau\rangle\right|_{z=1}=\frac{1-\langle y, \tau\rangle}{1-\left\langle U_{y} \tau, y\right\rangle}=\frac{|1-\langle y, \tau\rangle|^{2}}{1-\|y\|^{2}}>0 .
$$

Consequently, we have for $z \in \Gamma_{k}$,

$$
\frac{|1-\langle m(z \tau), \tau\rangle|}{1-\|m(z \tau)\|^{2}}=L \frac{|1-z|^{2}}{1-|z|^{2}} \cdot \frac{1}{|1-\langle m(z \tau), \tau\rangle|}<L k \frac{|1-z|}{|1-\langle m(z \tau), \tau\rangle|} .
$$

Since $\lim _{z \rightarrow 1}\left|\frac{1-\langle m(z \tau), \tau\rangle}{1-z}\right|=L$, it follows that if $z \in \Gamma_{k}$ is close enough to 1 , then $m(z \tau)$ is in $D_{\alpha}(\tau)(\alpha>k)$. Hence, by hypothesis (3) of the theorem, $\angle \lim _{z \rightarrow 1} \frac{f(m(z \tau))}{\|m(z \tau)-\tau\|^{3}}=0$.

Therefore equality (8) implies that $\angle \lim _{z \rightarrow 1} \frac{g(z)}{|z-1|^{3}}=0$, and by Proposition 2, $g \equiv 0$ on $\Delta$. So, by (7),

$$
\left\langle f(m(z \tau)),\left[M_{y}^{\prime}(m(z \tau))\right]^{*} U_{y} \tau\right\rangle=0 \quad \text { for all } \quad z \in \Delta .
$$

In particular, this equality holds for $z=0$; i.e.,

$$
\left\langle f(y),\left[M_{y}^{\prime}(y)\right]^{*} U_{y} \tau\right\rangle=0 \quad \text { for each } \quad y \in \mathbb{B} .
$$


By direct calculations, one obtains that

$$
\begin{aligned}
& M_{y}^{\prime}(x) h \\
& =\frac{1}{(1-\langle x, y\rangle)^{2}}\left[-(1-\langle x, y\rangle)\left(P_{y}+s Q_{y}\right) h+\langle h, y\rangle\left(y-P_{y} x-s Q_{y} x\right)\right] .
\end{aligned}
$$

Hence,

$$
M_{y}^{\prime}(y) h=-\frac{1}{1-\|y\|^{2}}\left(P_{y}+s Q_{y}\right) h
$$

and equality (9) is equivalent to

$$
\left\langle f(y),\left(P_{y}+s Q_{y}\right) U_{y} \tau\right\rangle=0 .
$$

Substituting

$$
U_{y} \tau=M_{y} \tau=\frac{y-P_{y} \tau-s Q_{y} \tau}{1-\langle\tau, y\rangle}
$$

in this equality, we obtain

$$
\left\langle f(y), y-\tau+\|y\|^{2} \tau-\langle\tau, y\rangle y\right\rangle=0 \quad \text { for all } \quad y \in \mathbb{B} .
$$

Let $y=y_{1} \tau+\tilde{y}$, where $y_{1}=\langle y, \tau\rangle$ and $\langle\tilde{y}, \tau\rangle=0$.

Similarly, $f(y)=f_{1}(y) \tau+\tilde{f}(y)$ with $f_{1}(y)=\langle f(y), \tau\rangle$ and $\langle\tilde{f}(y), \tau\rangle=0$ for all $y \in \mathbb{B}$.

Using this notation, we have

$$
\left\langle f_{1}(y) \tau, y_{1} \tau-\tau+\|y\|^{2} \tau-\left|y_{1}\right|^{2} \tau\right\rangle=-\left\langle\tilde{f}(y), \tilde{y}-\bar{y}_{1} \tilde{y}\right\rangle
$$

and

$$
\left(1-\bar{y}_{1}-\|\tilde{y}\|^{2}\right) f_{1}(y)=\left(1-y_{1}\right)\langle\tilde{f}(y), \tilde{y}\rangle .
$$

Differentiating this equality with respect to $\bar{y}_{1}$, we conclude that it can hold only if $f_{1}(y)=0$ and

$$
\langle\tilde{f}(y), \tilde{y}\rangle=0 \quad \text { for all } \quad y \in \mathbb{B} .
$$

Now let $\sigma$ be an arbitrary unit vector orthogonal to $\tau$, i.e., $\langle\sigma, \tau\rangle=0$. Suppose that $\tilde{y}=y_{2} \sigma+u$, where $y_{2}=\langle\tilde{y}, \sigma\rangle$ and $\langle u, \sigma\rangle=0$.

Similarly, $\tilde{f}(y)=f_{2}(y) \sigma+v(y)$ with $f_{2}(y)=\langle\tilde{f}(y), \sigma\rangle$ and $\langle v(y), \sigma\rangle=0$ for all $y \in \mathbb{B}$. Then by (10),

$$
f_{2}(y) \bar{y}_{2}=-\langle v(y), u\rangle
$$

Differentiating this equality with respect to $\bar{y}_{2}$, we obtain $f_{2}(y)=0$. Hence, $f \equiv 0$ on $\mathbb{B}$.

Following L. A. Harris 13 , we define the numerical range of each $h \in \operatorname{Hol}(\mathbb{B}, H)$ which has a norm continuous extension to $\overline{\mathbb{B}}$ by

$$
V(h):=\{\langle h(x), x\rangle:\|x\|=1\} .
$$

For an arbitrary holomorphic mapping $h \in \operatorname{Hol}(\mathbb{B}, H)$ and for each $s \in(0,1)$, we define the mapping $h_{s}: \frac{1}{s} \mathbb{B} \mapsto H$ by

$$
h_{s}:=h(s x), \quad\|x\|<\frac{1}{s}
$$

and set

$$
L(h):=\lim _{s \rightarrow 1^{-}} \sup \operatorname{Re}\left(V\left(h_{s}\right)\right)
$$


It is known (Theorem 1 in [14]) that the mapping $I-h$ is a generator if and only if $L(h) \leq 1$. So the following corollary is an immediate consequence of our theorem.

Corollary. Let $h \in \operatorname{Hol}(\mathbb{B}, H)$ with $L(h) \leq 1$. If for some $\tau \in \partial \mathbb{B}$, the $K$-limit

$$
K-\lim _{x \rightarrow \tau} \frac{h(x)-x}{\|x-\tau\|^{3}}=0,
$$

then $h \equiv I$ on $\mathbb{B}$

Since obviously $L(h) \leq 1$ for all self-mappings of $\mathbb{B}$, this corollary properly contains Proposition 3.

\section{REFERENCES}

[1] D. Aharonov, S. Reich and D. Shoikhet, Flow invariance conditions for holomorphic mappings in Banach spaces, Math. Proceedings of the Royal Irish Academy 99A (1999), 93-104. MR.1883068 (2002i:46038)

[2] H. Alexander, Holomorphic mappings from the ball and polydisc, Math. Ann. 209 (1974), 249-256. MR0352531 (50:5018)

[3] L. Baracco, D. Zaitsev and G. Zampieri, A Burns-Krantz type theorem for domains with corners, Math. Ann. 336 (2006), 491-504. MR2249756 (2007f:32021)

[4] E. Berkson and H. Porta, Semigroups of analytic functions and composition operators, Michigan Math. J. 25 (1978), 101-115. MR0480965 (58:1112)

[5] F. Bracci, R. Tauraso and F. Vlacci, Identity principles for commuting holomorphic self-maps of the unit disc, J. Math. Anal. Appl. 270 (2002), 451-473. MR1916591 (2003f:30030)

[6] D. M. Burns and S. G. Krantz, Rigidity of holomorphic mappings and a new Schwarz lemma at the boundary, J. Amer. Math. Soc. 7 (1994), 661-676. MR.1242454 (94j:32016)

[7] M. Elin, M. Levenshtein, S. Reich, and D. Shoikhet, Rigidity results for holomorphic mappings on the unit disk, in Complex and Harmonic Analysis, DEStech Publications, Lancaster, PA, 2007, 93-109.

[8] M. Elin, M. Levenshtein, D. Shoikhet, and R. Tauraso, Rigidity of holomorphic generators and one-parameter semigroups, Dynam. Syst. Appl. 16 (2007), 251-266. MR2330793

[9] M. Elin, S. Reich and D. Shoikhet, Complex dynamical systems and the geometry of domains in Banach spaces, Dissertations Math. 427 (2004). MR2071666 (2005g:47118)

[10] M. Elin and D. Shoikhet, Dynamic extension of the Julia-Wolff-Carathéodory theorem, Dynam. Syst. Appl. 10 (2001), 421-438. MR1858192 (2002j:30020)

[11] G. Gentili and S. Migliorini, A boundary rigidity problem for holomorphic mappings, Gen. Math. 5 (1997), 161-174. MR.1723606 (2000j:32027)

[12] K. Goebel and S. Reich, Uniform Convexity, Hyperbolic Geometry and Nonexpansive Mappings, Marcel Dekker, New York and Basel, 1984. MR744194 (86d:58012)

[13] L. A. Harris, The numerical range of holomorphic functions in Banach spaces, Amer. J. Math. 93 (1971), 1005-1019. MR0301505 (46:663)

[14] L. A. Harris, S. Reich and D. Shoikhet, Dissipative holomorphic functions, Bloch radii, and the Schwarz lemma, J. Analyse Math. 82 (2000), 221-232. MR.1799664 (2001k:46074)

[15] X. J. Huang, A boundary rigidity problem for holomorphic mappings on some weakly pseudoconvex domains, Canad. J. Math. 47 (1995), 405-420. MR.1335086 (96f:32041)

[16] E. Kopecká and S. Reich, Hyperbolic monotonicity in the Hilbert ball, Fixed Point Theory Appl. 2006, Article ID 78104, 1-15. MR2210916 (2007c:47063)

[17] M. Levenshtein, S. Reich and D. Shoikhet, An application of the resolvent method to rigidity theory for holomorphic mappings, J. Nonlinear Convex Anal. 8 (2007), 99-103. MR2314669

[18] S. Reich and D. Shoikhet, Semigroups and generators on convex domains with the hyperbolic metric, Atti. Accad. Naz. Lincei 8 (1997), 231-250. MR1631605 (99g:47155)

[19] S. Reich and D. Shoikhet, Nonlinear Semigroups, Fixed Points, and Geometry of Domains in Banach Spaces, Imperial College Press, London, 2005. MR2022955 (2006g:47105)

[20] W. Rudin, Function Theory in the Unit Ball of $\mathbb{C}^{n}$, Springer, Berlin, 1980. MR601594 $(82 \mathrm{i}: 32002)$ 
[21] D. Shoikhet, Semigroups in Geometrical Function Theory, Kluwer, Dordrecht, 2001. MR:1849612 (2002g:30012)

[22] R. Tauraso and F. Vlacci, Rigidity at the boundary for holomorphic self-maps of the unit disk, Complex Variables Theory Appl. 45 (2001), 151-165. MR.1909431 (2003e:30039)

Department of Mathematics, OrT Braude College, P.O. Box 78, 21982 Karmiel, ISRAEL

E-mail address: mark.elin@gmail.com

Department of Mathematics, The Technion - Israel Institute of Technology, 32000 HAIFA, IsRael

E-mail address: marlev@list.ru

Department of Mathematics, The Technion - Israel Institute of Technology, 32000 HaIfa, ISRAEL

E-mail address: sreich@tx.technion.ac.il

Department of Mathematics, OrT Braude College, P.O. Box 78, 21982 Karmiel, ISRAEL

E-mail address: davs27@netvision.net.il 